\title{
Perioperative dissemination of salmonella
}

\author{
M UHARI, H RANTALA, AND E HERVA \\ Department of Paediatrics, University of Oulu and the National Public Health Institute, Oulu, Finland
}

SUMMARY A hypophyseal adenoma was operated on in a patient who was a carrier of Salmonella typhimurium. A chronic salmonella osteitis developed in her calvarium at the site of operation. Our case and reported experience emphasise that asymptomatic carriers of salmonella should be given perioperative antibiotic prophylaxis, especially if they are immunosuppressed.

The clinical presentation of salmonella infections is variable, particularly in patients having operations and cytotoxic treatment. Wolfe et al found that the risk of salmonella infection was high, particularly in patients with leukaemia and lymphoma. ${ }^{1}$ Salmonella typhimurium and Salmonella enteritidis both cause bone and joint infections. ${ }^{2}$

In addition to having an increased risk of unusual complications associated with acute salmonella infections, carriers of salmonella receiving cytotoxic treatment should be regarded as having an additional preoperative risk factor. To emphasise this aspect of salmonella infection we report the case history of a girl with a hypophyseal adenoma who was operated on while she was a carrier of $S$ typhimurium. A chronic salmonella osteitis developed in her calvarium at the site of operation.

\section{Case report}

In September 1985 a 12 year old girl was referred to this department with a one week history of headache and right sided ptosis. Computed tomography was done, which showed changes consistent with a hypophyseal tumour. A few days before the onset of neurological symptoms she had had diarrhoea, as had other members of her family. Stool cultures from the patient and from other members of her family were positive for $S$ typhimurium. A craniotomy was performed. At operation the tumour was found to be inoperable but a biopsy specimen was taken. Histological examination showed the presence of an hypophyseal adenoma. Dexamethasone was given to decrease intracranial pressure and anticonvulsant treatment using phenytoin was started postoperatively. Three weeks after operation she had a course of radiotherapy. Recovery from the operation was uneventful
Table Timing and duration of drug treatment

\begin{tabular}{|c|c|c|}
\hline Drug & $\begin{array}{l}\text { Days after } \\
\text { operation that } \\
\text { drug started }\end{array}$ & $\begin{array}{l}\text { Duration } \\
\text { of } \\
\text { course }\end{array}$ \\
\hline Dexamethasone & -6 & 20 \\
\hline Phenytoin & 0 & Continuous \\
\hline \multirow{2}{*}{$\begin{array}{c}\text { Amphicillin (intravenously) } \\
\text { (orally) }\end{array}$} & 39 & 5 \\
\hline & 44 & 16 \\
\hline Trimethoprim/sulphamethoxazole & 66 & 17 \\
\hline Chloramphenicol (topically) & 74 & 18 \\
\hline \multicolumn{3}{|l|}{ Trimethoprim/sulphamethoxazole } \\
\hline (orally) & 164 & 9 \\
\hline (intravenously) & 173 & 16 \\
\hline (orally) & 189 & 8 \\
\hline
\end{tabular}

without signs or symptoms of systemic or local infection.

One week after the operation stool culture was still positive for $S$ typhimurium, whereas the stool samples taken during the subsequent six months were all negative. Four weeks postoperatively inflammation, swelling, and a small fluctuant mass were noted at the surgical wound site. Needle aspiration was performed and $S$ typhimurium cultured from the sample. The infection was unsuccessfully treated with several courses of antimicrobial drugs (table) to which the strain was sensitive on culture, the cultures from the wound remained positive.

Four and a half months after operation osteitic changes were seen on the skull $x$-ray picture and a further craniotomy performed. At operation it was found that the bone which in the primary operation had been transplanted to the calvarium from the iliac crest was infected. The infected piece of bone was removed. Before the second operation oral trimethoprim-sulphamethoxazole was given, and this was continued intravenously for 16 days after operation and then for a further eight days orally. After the second operation the wound healed, and no residual infection was detected during the 10 month follow up.

\section{Discussion}

There is a wide variety of complications of infections caused by any strain of salmonella. ${ }^{2}{ }^{3}$ The possibility of the development of bone infection in an asymp- 
tomatic carrier is not, however, well recognised. Gilman et al found that bone marrow is a more sensitive culture medium for Salmonella typhi than stool, blood, or urine in patients with typhoid fever. ${ }^{4}$ The reason for our patient developing bone infection may be due to the ability of salmonellas to invade bone marrow. If this is the case then the bacteria were actually in the bone marrow at the time of the primary operation. Another possibility is that occult bacteraemia may have occurred during the early postoperative period when the patient was still carrying salmonella. Young patients with positive blood cultures may be asymptomatic. ${ }^{5}$

The first sample from which $S$ typhimurium was cultured was taken by aseptic needle aspiration. The stool cultures had been negative for three weeks before signs of the infection at the operation site were detected which makes faecal contamination of the wound an unlikely source of infection. Our case corroborates the fact that salmonellas tend to infect diseased or injured tissue. The use of radiotherapy in conjunction with dexamethasone probably increased the likelihood of salmonella causing the infection.

Rodriguez et al recently published a review of intracranial infections caused by salmonellas. ${ }^{6}$ In their report one patient developed a focal salmonella infection in a neoplastic lesion within the central nervous system. Our patient did not have any symptoms or signs of intracranial infection, and was cured after the removal of the infected bone.

Our case shows that even an asymptomatic salmonella carrier should be given prophylaxis when any surgical operation is planned. Chloramphenicol or trimethoprim-sulphamethoxazole should be given before, during, and after the operation to prevent local complications. There is, however, no curative treatment for the salmonella carrier, and antimicrobial treatment may prolong carriage. The use of bone marrow culture for salmonella is a useful diagnostic sampling medium.

\section{References}

' Wolfe MS, Armstrong D, Louria DB, Blevins A. Salmonellosis in patients with neoplastic disease. A review of 100 episodes at Memorial Cancer Center over a 13 year period. Arch Intern Med 1971;128:546-54

2 Ortiz-Neu C, Marr JS, Cherubin CE, Neu HC. Bone and joint infections due to Salmonella. J Infect Dis 1978;138:820-8.

${ }^{3}$ Saphra I, Winter JW. Clinical manifestations of salmonellosis in man. An evaluation of 7779 human infections identified at the New York Salmonella Center. N Engl J Med 1957;256: 1128-34.

${ }^{4}$ Gilman RH, Terminel M, Levine MM, Hernandez-Mendoza P, Hornick RB. Relative efficacy of blood, urine, rectal swab, bone-marrow, and rose-spot cultures for recovery of salmonella typhi in typhoid fever. Lancet 1975;ii:1211-3.

5 Raucher HS, Eichenfield AH, Hodes HL. Treatment of salmonella gastroenteritis in infants. Clin Pediatr 1983;22:601-4.

${ }^{6}$ Rodriguez RE, Valero V, Watanakunakorn C. Salmonella focal intracranial infections: review of the world literature (1884 1984) and report of an unusual case. Rev Infect Dis 1986;8: $31-41$

Correspondence to $\mathrm{Dr} \mathrm{M}$ Uhari, Department of Paediatrics, University of Oulu, SF-90220 Oulu, Finland.

Received 13 May 1987 